Meta

Journal des tradlucteurs

Translators' Journal

\title{
Réflexions sur la néologie
}

\section{Jean Darbelnet}

Volume 17, numéro 2, juin 1972

URI : https://id.erudit.org/iderudit/002475ar

DOI : https://doi.org/10.7202/002475ar

Aller au sommaire du numéro

Éditeur(s)

Les Presses de l'Université de Montréal

ISSN

0026-0452 (imprimé)

1492-1421 (numérique)

Découvrir la revue

Citer cet article

Darbelnet, J. (1972). Réflexions sur la néologie. Meta, 17(2), 87-93.

https://doi.org/10.7202/002475ar d'utilisation que vous pouvez consulter en ligne.

https://apropos.erudit.org/fr/usagers/politique-dutilisation/ 


\section{Réflexions sur la néologie*}

Vox populi, vox Dei. Il est très tentant d'appliquer cet aphorisme à la néologie. Ce que le peuple fait en matière de langage est bien fait, et depuis Malherbe on ne saurait aller là contre. Au colloque sur la néologie tenu à Paris en mai 1971, M. Quemada n'a pas manqué de signaler la part d'imprévu et d'arbitraire qui entre dans l'adoption ou le rejet de mots nouveaux proposés aux usagers. Ce qui revient à dire que ce ne sont pas les termes les mieux conçus qui l'emportent nécessairement. L'usager, quand il se prononce, n'a pas forcément raison, mais on ne raisonne pas avec l'usage. Idéalement, un néologisme devrait répondre à une double exigence : d'une part être conforme au système linguistique dans lequel il est introduit, et d'autre part emporter l'adhésion de ceux qui sont appelés à l'employer. En fin de compte, c'est sans doute cette seconde exigence qui est déterminante. Le plus souvent, la chose se décide empiriquement, et, comme l'a fait observer M. Deroy, «quand une langue a besoin d'un mot, elle s'en accommode ou elle l'accommode».

Tout cela est difficile à contester. Cependant d'autres facteurs peuvent entrer en ligne de compte, à commencer par la centralisation administrative à laquelle il est très difficile d'échapper aujourd'hui. L'administration, parmi ses pouvoirs, a celui de la diffusion, qui lui permet, du moins officiellement, d'imposer le terme qu'elle décide de diffuser. C'est ainsi que, pendant la guerre de 1914-1918, il a suffi d'un ordre du général Estienne pour remplacer le mot tank par char d'assaut. De semblable façon s'est introduit dans l'usage le mot oléoduc qui, lui par contre, n'a pas réussi à évincer son concurrent pipe-line.

On peut, dans cet ordre d'idée, parler de dirigisme, même si ce terme n'est pas très populaire. Mais l'effort néologique suppose un choix, une recommandation, et naturellement le désir de faire accepter ce qui est proposé, et au besoin d'invoquer pour cela l'appui de certains organismes qui possèdent ce pouvoir de

* Ces réflexions ont été inspirées en grande partie par les débats du colloque sur la néologie qui s'est tenu à Paris en mai 1971 sous les auspices du Conseil international de la langue française. 
diffusion. Car on a toujours le droit de supposer que si la réaction du public est défavorable, elle peut n'être que passagère. Ainsi, oléoduc a été lancé en 1959 par l'administration de la canalisation de Hassi-Messaoud-Bougie, sur l'intervention d'un sénateur, M. Marcel Plaisant. Si, comme cela semble être le cas, cette tentative n'a obtenu qu'un succès partiel, on peut estimer que la cause est entendue et que pipe-line reste maître du terrain. Mais il n'est pas indifférent de faire ce genre d'essai, et on peut regretter que la S.N.C.F., pour des raisons d'ordre matériel d'ailleurs fort discutables, ne collabore pas à ce genre d'expérience en remplaçant, dans ses publications, car-ferry par traversier et container par conteneur qui ont l'avantage de donner l'un et l'autre une forme authentiquement française à des réalités nouvelles.

Cela revient, évidemment, à orienter l'usage, à s'assurer que certaines créations ne bénéficient pas d'un engouement passager. Motoneige s'est implanté au Canada sans qu'on ait pris le temps de se demander si motoluge n'eût pas été préférable, ne fût-ce que parce que ce mot se prêtait mieux à la dérivation : motoluger, motolugeur. Motoneigiste existe déjà, mais motoneiger, encore à venir, paraîtra curieux tant qu'on aura conscience du sens des éléments qui composent ce verbe. La raison alléguée que luge est un mot inconnu au Canada n'est pas convaincante. Tout le monde est appelé aujourd'hui à assimiler chaque année un certain nombre de mots nouveaux. Plus valable est l'argument invoquant l'existence du canadianisme autoneige qui ouvre la voie à motoneige. Le parallélisme qui en résulte est évidemment séduisant pour l'esprit.

De la même façon, il serait souhaitable, s'il en est encore temps, de rationaliser la forme des verbes dérivés de mythe et celle de leurs substantifs verbaux. Ainsi deux dérivés sont en concurrence pour exprimer la suppression d'un mythe : démythifier et démythiser. Il est évident que démythifier prête à confusion avec démystifier. De ce point de vue démythiser serait préférable, et il est d'ailleurs employé, mais moins fréquemment, semble-t-il, que son concurrent. Cependant le suffixe -iser est aujourd'hui plus vivant que -ifier. On peut se demander si, laissés à eux-mêmes, les usages sauront choisir entre les deux formes.

Le besoin d'exercer un contrôle sur les néologismes apparaît dans le cas de «décagénaire » qui, à première vue, se présente comme un équivalent ingénieux de teenager. Le mot est facile à prononcer et il semble s'inscrire dans une série, mais justement c'est là qu'il achoppe, comme l'a montré $M$. André Goosse dans ses chroniques de langage. Déca- veut dire dix, et en bonne logique, si le sexagénaire a entre soixante et soixante-neuf ans, le « décagénaire» serait plus que centenaire. Il suffit de réfléchir pour s'en rendre compte, mais la création de nouveaux vocables se fait à un tel rythme qu'on n'a pas le temps de les soumettre à une chambre de réflexion, dont la composition et les pouvoirs seraient d'ailleurs difficiles à fixer.

Le choix judicieux des néologismes suppose évidemment que l'on sait accepter aussi bien que proscrire. On a critiqué l'emploi du terme sous-médicalisé à l'égard d'un pays ou de sa population. Il est facile à ce propos de parler de jargon. Mais une saine politique du langage doit faire la part du jargon et reconnaître que les spécialistes ne peuvent pas toujours parler comme tout le monde. Si nous trouvons naturel, comme cela semble être le cas, de dire qu'une population 
est sous-scolarisée, il semble difficile d'exclure sous-médicalisé, qui décrit un phénomène parallèle. Il peut $\mathrm{y}$ avoir insuffisance de dispensaires, de centres de consultation aussi bien que d'établissements scolaires. Morphologiquement, ce composé est satisfaisant; sémantiquement, il suppose l'existence d'un verbe «médicaliser » voulant dire « équiper en moyens médicaux». Un tel verbe peut surprendre au premier abord, mais il est parfaitement concevable. Si on l'adopte, on sera alors amené à parler de «médicalisation» pour désigner l'accès d'une population aux soins médicaux dont elle a besoin, ou, si l'on préfère, la diffusion des moyens médicaux au sein de cette population. Dans une langue assez technique, il paraîtra alors normal de dire (et peut-être le dit-on déjà), à propos d'un pays en voie de développement, que sa médicalisation progresse plus lentement (ou plus rapidement) que sa scolarisation. Ce sont là de nouvelles préoccupations sociales que la langue doit s'efforcer de rendre.

Si l'on réfléchit aux moyens dont on dispose pour augmenter le vocabulaire, on s'aperçoit qu'ils sont peu nombreux. On peut partir de zéro, comme ce fut le cas avec gaz et comme cela l'est aujourd'hui pour les noms de marque, ou transformer des noms propres (noms de personnes et noms de lieux) en noms communs. Mais le plus souvent on emprunte, ou l'on part d'un mot existant pour lui donner un dérivé ou un composé. Il existe encore deux autres ressources, la néologie syntagmatique (ou formation de nouveaux syntagmes, facilement assimilables à des composés) et la néologie sémantique (addition de sens nouveaux à des mots déjà existants).

L'emprunt est généralement externe. Il porte alors sur les mots d'une langue étrangère. Il peut être aussi interne, et dans ce cas on remet en activité des mots sortis de l'usage, ou l'on puise dans le vocabulaire des parlers régionaux. Il semble que l'on soit loin d'avoir tiré tout le parti possible de cette seconde forme d'emprunt. Qu'il soit voulu ou accidentel, ce mode de recrutement est peu développé. On peut citer, à titre d'exemple, le vieux mot foresterie qui, il y a quelques années, s'est introduit dans la désignation d'une faculté de l'Université Laval. Pour ce qui est des régionalismes, le cas de rescapé, entré dans le français commun à la suite d'une catastrophe, est bien connu ; on peut lui opposer celui de congère, dont la diffusion s'est faite, par contre, insensiblement.

M. Deroy a distingué les emprunts nécessaires, qui comblent des lacunes, et les emprunts de luxe qui sont souvent dus au snobisme et doublent des mots existants. Certains estimeront, par exemple, que nous pouvons nous passer de travelers et de charters. Ce n'est pas l'avis des agences de voyage. Ici encore intervient la notion de niveau de langue, et ces deux anglicismes peuvent être considérés comme faisant d'abord partie d'un certain argot de métier. Il est fort possible qu'ils aient un jour le même sort que high life et bank-note, aujourd'hui tombés dans l'oubli.

Il existe contre les emprunts un préjugé très fort qui ne tient pas compte du fait que les langues ont toujours emprunté, plus ou moins. L'emprunt lexical ne devient inquiétant que lorsqu'il introduit un nombre très élevé de mots étrangers, et c'est là une question d'appréciation. L'emprunt syntaxique est considéré comme plus dangereux, mais il est rare. Un dirigisme bien compris essaiera de freiner les emprunts de luxe et accueillera au contraire ceux qui comblent des lacunes. 
Le français n'assimile plus comme il l'a fait aux siècles passés (cf. paquebot et redingote). Il n'en reste pas moins que le mot étranger doit être facile à prononcer, c'est-à-dire s'insérer dans le système phonologique du français. Quand la traduction du roman de Sterne, $A$ Sentimental Journey, a introduit en 1769 l'adjectif sentimental, son adoption a été d'autant plus aisée qu'il avait l'air d'un mot français et pouvait se prononcer comme tel. En fait, rares sont ceux qui savent que ce mot a été un anglicisme. L'adoption de motel et, à un niveau différent, de self, s'explique de la même façon. Par contre, la résistance que l'on oppose au terme design se justifie en grande partie par la difficulté qu'on éprouve à donner à ce mot une prononciation française qui ne s'écarte pas trop de son orthographe. On sait que notre langue répugne aujourd'hui à changer l'orthographe des mots qu'elle emprunte.

«Le français, a dit Jules Marouzeau, est peut-être une des langues les plus lacuneuses. »Il est certain que malgré ses préfixes et ses suffixes, il offre par rapport à l'anglais et à l'allemand de nombreuses lacunes de dérivation. De plus il ne forme pas des composés avec la même facilité. À ces déficiences d'ordre structural s'ajoute, de la part des usagers, une grande hésitation à former des mots nouveaux à partir de mots déjà existants. Le provignement est bridé par ce qu'on a appelé avec raison le malthusianisme, et nous avons cité à ce propos l'exemple de sous-médicalisé. Il semble avéré que le français pourrait faire un meilleur usage de ses ressources dans ce domaine, même si elles sont moindres que dans d'autres langues.

L'observation des faits devrait pourtant nous inciter à plus de hardiesse. Le terme décolonisation n'est apparu dans les dictionnaires que vers 1960, alors que le processus qu'il désigne était amorcé depuis plusieurs années. Disons que décolonisation a été une virtualité de la langue française qui a fini par devenir une réalité. Il en est de même de assurabilité qu'il ne faut pas hésiter à accepter si on en a besoin (il n'est pas encore dans le G.L.L.F. paru en 1971). Il y a ainsi des virtualités qui attendent qu'un besoin se manifeste pour se muer en réalités. Il se peut que parapher continue à suffire à nos besoins d'expression, et dans ce cas il est inutile d'innover. Mais rien ne s'opposerait à ce que naisse le verbe initialer, si le besoin d'une distinction ou la généralisation d'un certain usage administratif justifiait cette création qui, morphologiquement parlant, est irréprochable.

Mais, pour réaliser les virtualités de la langue, il faudrait se débarrasser d'idées surannées et d'un certain impressionnisme. Par exemple, il n'est plus possible aujourd'hui de refuser la combinaison d'un élément grec avec un élément latin. On oublie d'ailleurs que dès 1820 la formation de autoclave a enfreint cette prétendue règle, bien avant que la création du mot automobile ne provoque une longue discussion à ce sujet. Depuis il y a eu monoplan, biplan, autofinancement, etc., pour aboutir à reprographie. Par ailleurs nous avons trop tendance à juger les mots nouveaux d'après une première impression que l'habitude ne manquerait pas de corriger dans bien des cas. En quoi écographie est-il plus condamnable que géographie? Est-il bien sûr que associativité, communativité, distributivité heurtent l'esthétique de la langue? Il est bien évident que certains mots en -tion demandent 
à être doublés d'une forme en -tivité qui permet de marquer une nuance importante. Ainsi nous sommes obligés de distinguer entre production et productivité.

Au début la langue fourche, mais c'est une affaire d'habitude. Il s'est trouvé des gens instruits vers 1945 pour achopper sur la prononciation d'un terme alors nouveau : existentialisme. On prête parfois aussi aux néologismes un caractère comique qu'il est difficile de justifier objectivement. Ainsi d'oléoduc on a dit que cela faisait penser à grand-duc et à archiduc, mais cette plaisanterie ne s'applique apparemment pas à viaduc et à aqueduc, logiquement tout aussi vulnérables.

Il y aurait, comme le propose l'auteur anonyme d'un document sur la néologie préparé pour le C.I.L.F. en 1970, un travail de réfection à faire dans ce domaine ; il consisterait à compléter des séries de dérivés, non pas gratuitement mais là où le besoin d'un nouveau terme se ferait sentir. En particulier, il serait bon de faire artificiellement ce que l'histoire a fait avec des mots latins qui sont beaucoup moins bien représentés en français qu'en anglais. Dans le français de tous les jours, le verbe latin trudere ne survit guère que dans intrusion et intrus : il est par contre le radical d'une dizaine de mots anglais d'un usage courant. Dans cette perspective, on peut même se demander si nous devrions nous refuser la commodité de matur et immatur que les psychologues emploient déjà et qui existent en anglais. À en juger par le dictionnaire des rimes de P. Desfeuilles, le français a très peu d'adjectifs en -ur. Rien n'empêcherait de franciser, au fur et à mesure des besoins, des adjectifs latins en -urus.

Si le français n'a pas la facilité de l'anglais et surtout de l'allemand pour former des composés où le déterminant précède le déterminé, la voie lui est largement ouverte pour procéder par juxtaposition d'un déterminé et de son déterminant. C'est le modèle, largement utilisé en terminologie, de cas limite, assurance-incendie, wagon-restaurant, etc.

Il y a lieu d'élargir la notion de composé, de ne pas la restreindre aux mots réunis par des traits d'union, du type abat-jour ou garde-chasse que l'on trouve dans les grammaires à propos du pluriel. Pêcher à la ligne est en fait un composé, et il en est de même de mise en route, mise en service, où les seconds substantifs sont des virtuels, ainsi que des combinaisons nom + adjectif ou participe passé, du type carte perforée, bandes dessinées. C'est ce que l'on appelle la néologie syntagmatique. Elle se heurte toutefois à l'objection qu'un seul mot vaut mieux que deux, et on peut se demander si le désir d'aller vite, poussé jusqu'à l'obsession, n'a pas le fâcheux effet de limiter l'exploitation d'une ressource importante.

Si l'on propose, par exemple, de faire imprimer en petit caractère sur l'étui d'une aiguille à injection les mots "pour un seul usage», on vous objectera que c'est trop long. En fait, cette expression n'est pas plus longue que le substantif incommensurabilité et à peine plus longue que l'adverbe individuellement, ce qui laisse à penser que ce n'est pas tant la brièveté du terme que l'on recherche que son caractère unitaire. L'adjectif jetable, que semblent avoir adopté les médecins français, a l'avantage de pouvoir se substantiver et aussi de servir de générique pour toutes sortes d'objets qu'on jette après un seul usage. C'est un trait de notre civilisation post-industrielle, où l'abondance de la matière va de pair avec le coût élevé de la main-d'œuvre, qu'elle préfère remplacer plutôt que de réparer ou d'entretenir. 
Le fétichisme du terme unitaire explique en partie la préférence des spécialistes pour design et leur éloignement de esthétique industrielle, terme qui pourrait d'ailleurs être remplacé, pour tenir compte de l'évolution de cette technique, par création industrielle.

À la néologie syntagmatique vient s'ajouter ce que l'on pourrait appeler la néologie sémantique, c'est-à-dire l'adjonction de sens nouveaux à des mots déjà existants. Parce que les mots français sont peu motivés, il leur est facile de posséder un grand nombre de sens, et la polysémie peut être considérée comme une caractéristique du français et une ressource dont les «néologues » auraient tort de de se priver. Il y a des emprunts sémantiques aussi bien que lexicaux, et l'emprunt sémantique consiste à donner à un mot français un sens que possède un mot étranger généralement apparenté. C'est le cas de promotion dans "promotion des ventes » et de procédure employé en dehors de la langue judiciaire. Certains vont jusqu'à parler de réhabilitation au sens de réadaptation ou de rééducation. Il convient de noter que ce dernier anglicisme est inutile, puisque nous avons déjà un mot en français pour rendre l'une ou l'autre de ces deux idées.

Mais l'extension de sens peut ne rien devoir à une langue étrangère. Elle constitue alors un procédé particulièrement économique et presque invisible. C'est peut-être son manque de relief qui l'empêche d'être utilisée là où elle pourrait l'être. Il arrive qu'on cherche un mot nouveau alors qu'on pourrait fort bien utiliser un mot déjà existant en lui donnant un sens nouveau ou en lui rendant un sens qu'il avait perdu. On peut citer comme exemple de ce procédé l'emploi de frégate et de corvette pendant la dernière guerre. Ce n'est pas ainsi qu'on s'y est pris pour faire la relève de living-room. Quand, dans certains appartements modernes, le salon et la salle à manger ont été remplacés par une seule pièce, on a appelé celle-ci la salle de séjour. Cette expression s'est ensuite abrégée en séjour, et non en salle. Il eût été plus simple de revenir au vieux mot salle tel qu'il s'employait dans mainte demeure campagnarde. Mais à chose nouvelle, on préfère une appellation nouvelle, et on ne saurait exagérer l'influence de la publicité sur l'évolution de la langue. C'est peut-être pour cela que commercialisation a du mal à s'imposer en face de marketing, et que certains préfèrent management à gestion. On dira que ce n'est pas la même chose, mais, en fait, rien n'empêche des mots français d'acquérir les sens de leurs concurrents anglais; il suffirait de leur en laisser le temps et ce ne serait pas très long. On oublie que si week-end n'est plus remplaçable, en France, par fin de semaine, c'est à cause du réseau d'associations qui s'est tissé autour du mot anglais depuis qu'il a pris la place de la * semaine anglaise ». Il y a une sorte de sédimentation qui se fait dans le contenu des mots.

Si la néologie syntagmatique se heurte au fétichisme du terme unitaire, la néologie sémantique est contrariée par l'attrait du terme univoque. Au fond l'usager aspire à un état de langue où non seulement toutes les désignations seraient unitaires, c'est-à-dire exprimées par un seul mot, mais aussi où il n'y aurait qu'un sens par mot et qu'un mot pour chaque sens. C'est là un rêve irréalisable. N'empêche que l'on constate dans la masse un certain embarras quand il s'agit de choisir entre les sens d'un même mot, et une tendance à refuser un mot du fait qu'un de ses sens est péjoratif. Il y a au Canada des gens qui n'osent pas employer le verbe exploiter à cause de l'exploitation de l'homme par l'homme. 
C'est pour des raisons du même ordre que l'adjectif inférieur a disparu de l'appellation de trois départements français.

Nous sommes ainsi ramenés à la deuxième exigence mentionnée au début de cet article : il ne suffit pas que le mot soit bien formé, il faut qu'il soit accepté. Il y aurait une étude à faire des facteurs psychologiques qui font obstacle à la rationalisation de la néologie.

JEAN DARBELnet 